\title{
Yukawa's of light stringy states (at D-brane intersections)
}

\section{Pascal Anastasopoulos*}

Universität Wien - Theoretische Physik Boltzmanngasse 5, A-1090 Vienna, Austria

E-mail: pascal.anastasopoulos@univie.ac.at

\section{Massimo Bianchi}

Dipartimento di Fisica, Università di Roma "Tor Vergata" \&

I.N.F.N. Sezione di Roma "Tor Vergata”, Via della Ricerca Scientifica, 00133 Roma, Italy

E-mail: massimo.bianchi@roma2.infn.it

\section{Dario Consoli}

Dipartimento di Fisica, Università di Roma “Tor Vergata”, Via della Ricerca Scientifica, 00133

Roma, Italy

E-mail: dario.consolieroma2.infn.it

In [1], we use 4-point amplitudes initially to normalise the vertex operators for massless and massive strings living at D-brane intersections and next to evaluate Yukawa couplings between these states. Our results can be used in order to evaluate physical processes which might be visible at LHC.

Corfu Summer Institute 2017 "School and Workshops on Elementary Particle Physics and Gravity" 2-28 September 2017

Corfu, Greece

${ }^{*}$ Speaker. 


\section{Introduction}

D-brane string vacua has been proven to be a very successful framework for semi-realistic model building ${ }^{1}$. Open strings stretched between stacks of some lower-dimensional hyperplanes, called D-branes, describe gauge fields (when the two endpoints end on the same stack of branes) and chiral matter (when the endpoints end on different stacks). Therefore, all Standard Model fields can be described by open strings while gravity is mediated by closed strings ${ }^{2}$.

A key feature of these vacua is that the string scale can be very low, even at a few $\mathrm{TeV}$ range [26-28], and stringy effects become possible candidates for physics beyond the Standard Model (see for example anomalous $Z^{\prime}$ [29-42], Kaluza Klein states [43-49], or purely stringy signatures $\left.[50-63]^{3}\right)$.

Another important prediction of all D-brane realizations of the Standard Model is that each matter field is followed by a whole tower of massive copies. That relays on the fact that a string living at the intersection of two different stacks of D-branes, vibrates with frequencies proportional to the angle between the branes $\theta$ generating a whole tower of massive modes with the same quantum numbers (realised by the endpoints). Therefore, the lowest modes on the intersections realise the electrons, the quarks, the Higgs etc and the highest modes as massive copies of them.

If the string scale $M_{s}$ is at a few $\mathrm{TeV}$ range and some angles between the branes are very small, these copies can be very light (light stringy states) and they might be visible in future experiments [66-69].

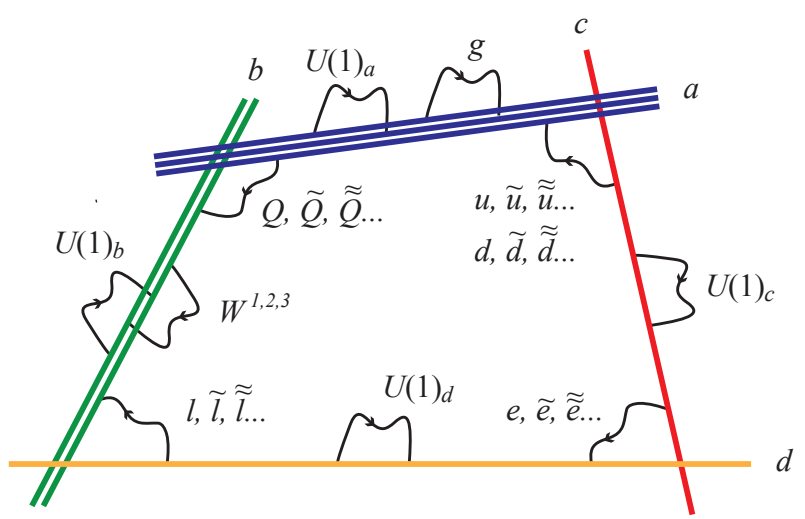

Figure 1: Local D-brane realization of the Standard Model. Each field is accompanied by a whole tower of massive excitations.

Even though the tower of massive stringy states looks like KK towers, there are differences. First of all the mass gaps at KK models are typically universal. In intersecting D-brane models each tower of states lives on different intersections with unique angle and therefore unique mass gap. Second the mass gaps in KK modes are typically proportional to the moding $(\sim m / R)$ whereas in intersecting D-brane models they are proportional to the square root of the moding $\left(\sim \sqrt{m} M_{s}\right)$.

\footnotetext{
${ }^{1}$ For original work, see [2-14] and for recent reviews on D-brane model building, see [15-19] and references therein.

${ }^{2}$ For original work on local D-brane configurations, see [20,21]. For a systematic analysis of local D-brane configurations, see [22-25].

${ }^{3}$ For recent reviews, see $[64,65]$.
} 
Finally, interactions which are prohibited due to conservation of internal momentum (for example the decay of an excited KK mode to two unexcited modes) are allowed in intersecting D-brane models is different from zero [1].

In [1] we compute Yukawa couplings between different stringy states of the towers living at D-brane intersections. We first evaluate 4-point amplitudes and by factorization we first normalise our relevant vertex operators (VO) [70,71] and next we compute the Yukawa couplings. In this proceeding we sketch the procedure giving some key point of the evaluation. More details can be found in the original work [1].

The plan is as follows. In Section 2, we describe the set-up of intersecting D6-branes on tori and present the expressions for both massless and massive BRST invariant vertex operators. In Section 3 we compute scattering amplitudes on the disk (tree level). Normalization problems are solved by first considering amplitudes that expose vector boson exchange on one or both channels $(s$ and $t)$.

\section{Setup and vertex operators}

Consider three stacks of D6-branes wrapping factorizable 3-cycles on a six-torus $T^{6}=T^{2} \times$ $T^{2} \times T^{2}$, labeled by $a, b$ and $c$. In each torus $T_{I}^{2}(I=1,2,3)$ two stacks $a$ and $b$ are intersecting at an angle $\theta_{a b}^{I}=\pi a_{a b}^{I}$ (next we will use the angles $a_{a b}^{I}$ subtracting $\pi$ for simplicity). Supersymmetry requires $\pm a_{a b}^{1} \pm a_{a b}^{2} \pm a_{a b}^{3}=0 \bmod 2$, for some choice of signs ${ }^{4}$. For non-vanishing Yukawa couplings, we take

$$
\begin{array}{lrrrr}
a_{a b}^{1}+a_{a b}^{2}+a_{a b}^{3}=0 & 0<a_{a b}^{1}<1 & 0<a_{b c}^{1}<1 & -1<a_{c a}^{1}<0 \\
a_{b c}^{1}+a_{b c}^{2}+a_{b c}^{3}=0 & 0<a_{a b}^{2}<1 & 0<a_{b c}^{2}<1 & -1<a_{c a}^{2}<0 \\
a_{c a}^{1}+a_{c a}^{2}+a_{c a}^{3}=-2 & -1<a_{a b}^{3}<0 & -1<a_{b c}^{3}<0 & -2<a_{c a}^{3}<0
\end{array}
$$

Strings ending on two different stacks of branes give rise to a massless (chiral) spectrum with multiplicity given by the number of intersections and a massive spectrum. The VO's for the lowest string modes (massless and first massive) living at intersections are given by [70]

$$
\begin{aligned}
& V_{\phi_{0}=\phi_{0}^{a b}}^{(-1)}=C_{\phi_{0}} e^{-\phi_{10}} \phi_{0} e^{-\varphi} \sigma_{a_{a, b}^{1}} \sigma_{a_{a, b}^{2}} \sigma_{1+a_{a, b}^{3}} e^{i\left[a_{a, b}^{1} \varphi_{1}+a_{a, b}^{2} \varphi_{2}+\left(a_{a, b}^{3}+1\right) \varphi_{3}\right]} e^{i k X} \\
& V_{\psi_{0}=\chi_{0}^{b c}}^{\left(-\frac{1}{2}\right)}=C_{\psi_{0}} e^{-\phi_{10}} \psi_{0}^{\alpha} S_{\alpha} e^{-\frac{\varphi}{2}} \sigma_{a_{b, c}^{1}} \sigma_{a_{b, c}^{2}} \sigma_{1+a_{b, c}^{3}} e^{i\left[\left(a_{b, c}^{1}-\frac{1}{2}\right) \varphi_{1}+\left(a_{b, c}^{2}-\frac{1}{2}\right) \varphi_{2}+\left(a_{b, c}^{3}+\frac{1}{2}\right) \varphi_{3}\right]} e^{i k X} \\
& V_{\chi_{0}=\chi_{0}^{c a}}^{\left(-\frac{1}{2}\right)}=C_{\chi_{0}} e^{-\phi_{10}} \chi_{0}^{\alpha} S_{\alpha} e^{-\frac{\varphi}{2}} \sigma_{1+a_{c, a}^{1}} \sigma_{1+a_{c, a}^{2}} \sigma_{1+a_{c, a}^{3}} e^{i\left[\left(a_{c, a}^{1}+\frac{1}{2}\right) \varphi_{1}+\left(a_{c, a}^{2}+\frac{1}{2}\right) \varphi_{2}+\left(a_{c, a}^{3}+\frac{1}{2}\right) \varphi_{3}\right]} e^{i k X} \\
& V_{\psi_{1}=\chi_{1}^{b c}}^{\left(-\frac{1}{2}\right)}=C_{\psi_{1}} e^{-\phi_{10}} \psi_{1}^{\alpha} S_{\alpha} e^{-\frac{\varphi}{2}} \tau_{a_{b, c}^{1}} \sigma_{a_{b, c}^{2}} \sigma_{1+a_{b, c}^{3}} e^{i\left[\left(a_{b, c}^{1}-\frac{1}{2}\right) \varphi_{1}+\left(a_{b, c}^{2}-\frac{1}{2}\right) \varphi_{2}+\left(a_{b, c}^{3}+\frac{1}{2}\right) \varphi_{3}\right]} e^{i k X} \\
& +C_{\tilde{\psi}_{1}} e^{-\phi_{10}} \tilde{\psi}_{1 \dot{\alpha}}^{\dagger} C^{\dot{\alpha}} e^{-\frac{\varphi}{2}} \sigma_{a_{b, c}^{1}} \sigma_{a_{b, c}^{2}} \sigma_{1+a_{b, c}^{3}} e^{i\left[\left(a_{b, c}^{1}+\frac{1}{2}\right) \varphi_{1}+\left(a_{b, c}^{2}-\frac{1}{2}\right) \varphi_{2}+\left(a_{b, c}^{3}+\frac{1}{2}\right) \varphi_{3}\right]} e^{i k X} \\
& V_{\chi_{1}=\chi_{1}^{c a}}^{\left(-\frac{1}{2}\right)}=C_{\chi_{1}} e^{-\phi_{10}} \chi_{1}^{\alpha} S_{\alpha} e^{-\frac{\varphi}{2}} \sigma_{1+a_{c, a}^{1}} \sigma_{1+a_{c, a}^{2}} \tau_{1+a_{c, a}^{3}} e^{i\left[\left(a_{c, a}^{1}+\frac{1}{2}\right) \varphi_{1}+\left(a_{c, a}^{2}+\frac{1}{2}\right) \varphi_{2}+\left(a_{c, a}^{3}+\frac{1}{2}\right) \varphi_{3}\right]} e^{i k X} \\
& +C_{\tilde{\chi}_{1}} e^{-\phi_{10}} \tilde{\chi}_{1 \dot{\alpha}}^{\dagger} C^{\dot{\alpha}} e^{-\frac{\varphi}{2}} \sigma_{1+a_{c, a}^{1}} \sigma_{1+a_{c, a}^{2}} \sigma_{1+a_{c, a}^{3}} e^{i\left[\left(a_{c, a}^{1}+\frac{1}{2}\right) \varphi_{1}+\left(a_{c, a}^{2}+\frac{1}{2}\right) \varphi_{2}+\left(a_{c, a}^{3}-\frac{1}{2}\right) \varphi_{3}\right]} e^{i k X}
\end{aligned}
$$

\footnotetext{
${ }^{4}$ Semi-realistic MSSM constructions on factorizable orbifolds can be found in [22,72-94].
} 

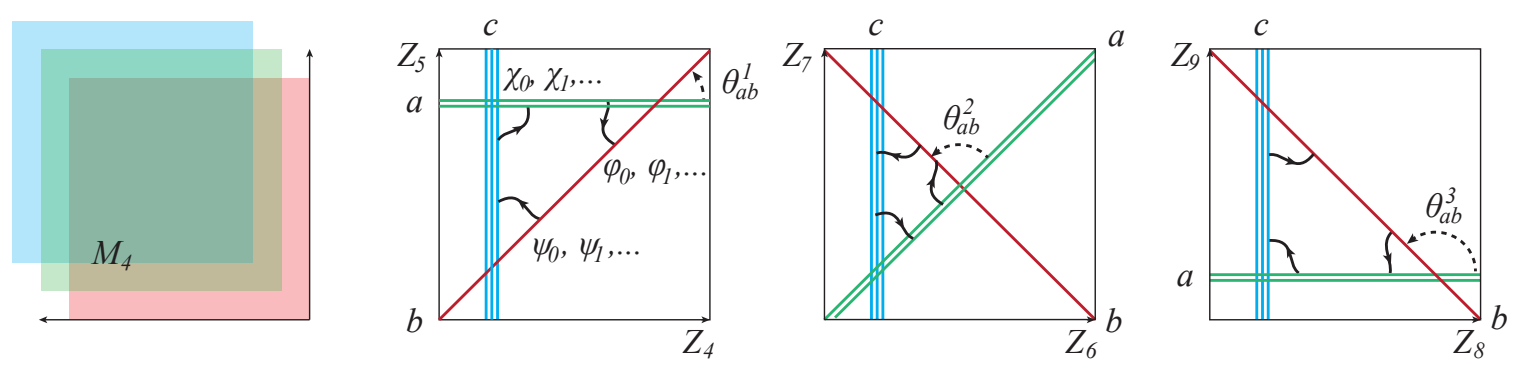

Figure 2: A simple configuration of three stacks of D6-branes in a torus $T_{I}^{2}$. The angles in the figure are large for illustrative purposes.

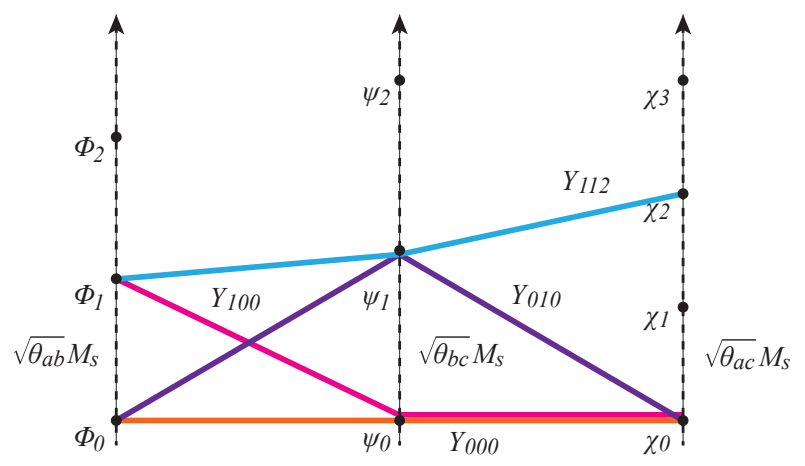

Figure 3: We evaluate Yukawas $Y_{i j k} \Phi_{i} \psi_{j} \chi_{k}$ where $i, j, k=0,1,2 \ldots$ the different massive modes, which are denoted by the lines connecting the different modes.

and the masses of the corresponding fields are

$$
\begin{aligned}
& \text { massless : } \quad m_{\phi_{0}}^{2}=0 \quad, \quad m_{\psi_{0}}^{2}=0 \quad, \quad m_{\chi_{0}}^{2}=0 \\
& \text { massive : } \quad, \quad m_{\psi_{1}}^{2}=a_{b c}^{1} / \alpha^{\prime}, \quad m_{\chi_{1}}^{2}=\left(1-\left|a_{c a}^{3}\right|\right) / \alpha^{\prime} .
\end{aligned}
$$

Having the expression for the VO's of the massless and massive states living at intersections, we evaluate string amplitudes and consequently the Yukawa couplings.

\section{Strategy}

Three-point amplitudes (Yukawas also) are ambiguous due to the unnormalised VO's of the incoming fields. Therefore, we have to start by four-point amplitudes and by factorisations normalise the corresponding VO's and then evaluate physical amplitudes.

In order to normalise our fields we evaluate their couplings to gauge fields and we equate them with the known values. With normalised VO's at hand we proceed to Yukawas. Schematically the strategy is given in the diagram 4 .

\subsection{The amplitude $\mathscr{A}\left(\bar{\psi}_{0}, \psi_{0}, \chi_{0}, \bar{\chi}_{0}\right)$}

Following the strategy above we compute specific 4-point amplitudes. Here, we present few examples, and more details can be found at our original work [1]. 


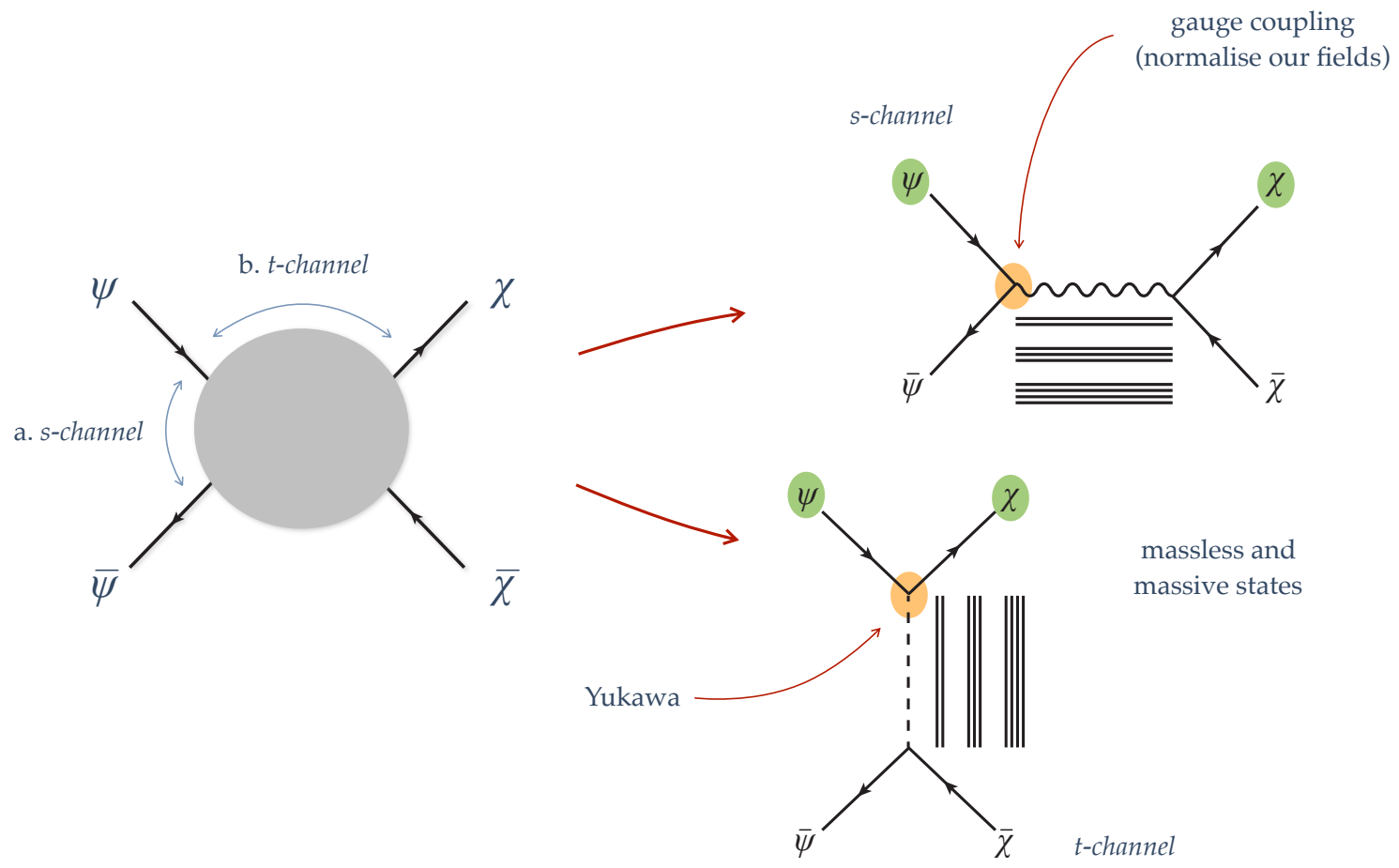

Figure 4: Factorization of the 4-point amplitude: a. At the lowest level of the s-channel we have the exchange of a gauge field which helps to normalise our fields. b. At t-channel we extract the various Yukawa couplings.

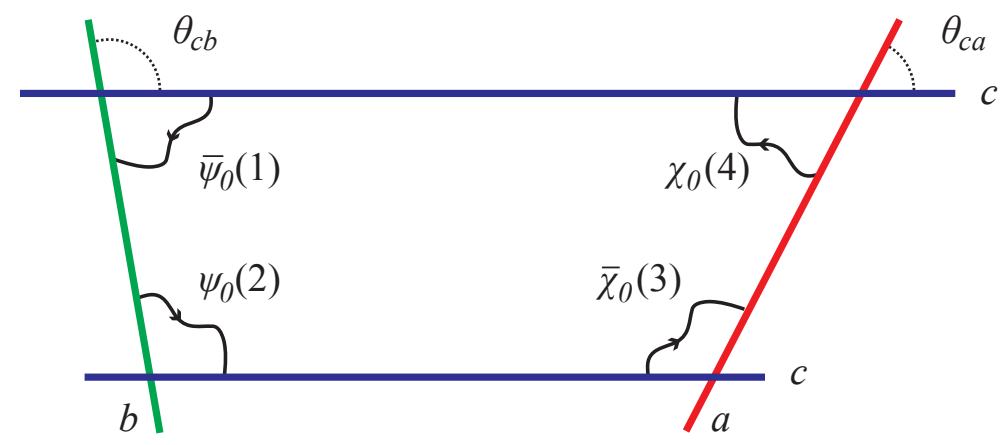

Figure 5: A configuration of branes describing the scattering between the spinors $\psi_{0}$ and $\chi_{0}$.

The amplitude $\mathscr{A}\left(\bar{\psi}_{0}, \psi_{0}, \chi_{0}, \bar{\chi}_{0}\right)$ with two-independent angles yields

$$
\begin{aligned}
\mathscr{A}\left(\bar{\psi}_{0}^{c b}, \psi_{0}^{b c}, \chi_{0}^{c a}, \bar{\chi}_{0}^{a c}\right)= & \left\langle V_{\bar{\psi}_{0}}^{\left(-\frac{1}{2}\right)} V_{\psi_{0}}^{\left(-\frac{1}{2}\right)} V_{\chi_{0}}^{\left(-\frac{1}{2}\right)} V_{\bar{\chi}_{0}}^{\left(-\frac{1}{2}\right)}\right\rangle \\
= & g_{\mathrm{op}}^{2} \alpha^{\prime} \psi_{0}(2) \cdot \chi_{0}(3) \bar{\psi}_{0}(1) \cdot \bar{\chi}_{0}(4) \int_{0}^{1} d x x^{\alpha^{\prime} s-1}(1-x)^{\alpha^{\prime} t-1} \times \\
& \times \prod_{I=1}^{3} \frac{4 \pi^{2} K_{I}^{c, a b} \alpha^{\prime}}{L_{a, I}^{1 / 4} L_{b, I}^{5 / 4} L_{c, I}^{1 / 2}} \frac{\sqrt{\alpha^{\prime}}}{L_{c, I} G_{1}^{(I)}(x)} \sum_{n_{I}, m_{I}} e^{-S_{\mathrm{Ham}}^{(I)}\left(m_{I}, n_{I}\right)}
\end{aligned}
$$

where $L_{a}, I$ is the brane $a$ 's length in torus $I . I_{a b}$ and similar are the number or intersections be- 
tween the branes on the torus. $G_{1}^{(I)}(x)$ are hypergeometric functions $G_{1}={ }_{2} F_{1}(\alpha, 1-\beta ; 1 ; z), G_{2}=$ ${ }_{2} F_{1}(1-\alpha, \beta ; 1 ; z), K_{I}^{c, a b}$ is an overall normalization constant and $S_{\mathrm{Ham}}^{(I)}\left(m_{I}, n_{I}\right)$ is the classical action in "hamiltonian" form:

$$
S_{\mathrm{Ham}}^{(I)}\left(m_{I}, n_{I}\right)=\frac{\pi t_{I}(x)}{\sin \pi\left|a_{b c}^{I}\right|}\left(\frac{4 \pi^{2} \alpha^{\prime} m_{I}^{2}}{L_{c, I}^{2}}+\frac{\sin ^{2} \pi\left|a_{b c}^{I}\right| L_{b, I}^{2}}{4 \pi^{2} \alpha^{\prime}} \frac{I_{c a, I}^{2} n_{I}^{2}}{\operatorname{gcd}^{2}\left(\left|I_{b c, I}\right|,\left|I_{c a, I}\right|\right)}\right)-2 \pi i \frac{m_{I}}{L_{c, I}} f_{\chi \psi, I}
$$

In the $s$ channel the amplitude shows a gauge boson propagating between parallel branes of type $c$, we can use the factorization in this channel to fix $K_{I}^{c, a b}=\frac{L_{a}^{1 / 4} L_{b}^{5 / 4} L_{c, I}^{1 / 2}}{(2 \pi)^{2} \alpha^{\prime}}$. The vertex operators' normalizations obtained from the amplitudes are

$$
\begin{gathered}
C_{A_{i}}=\sqrt{2 \alpha^{\prime}} \prod_{I}\left[\frac{\alpha^{\prime}}{L_{i, I}^{2}}\right]^{1 / 4} \\
C_{\chi_{0}^{i j}}=\left(\alpha^{\prime}\right)^{1 / 4} \sqrt{2 \alpha^{\prime}} \prod_{I}\left[\frac{\alpha^{\prime}}{L_{i, I} L_{j, I}}\right]^{1 / 4} \quad C_{\phi_{0}^{i j}}=\sqrt{2 \alpha^{\prime}} \prod_{I}\left[\frac{\alpha^{\prime}}{L_{i, I} L_{j, I}}\right]^{1 / 4} \\
C_{\chi_{1}^{i j}}=\left(\alpha^{\prime}\right)^{1 / 4} \sqrt{2 \alpha^{\prime}} \prod_{I}\left[\frac{\alpha^{\prime}}{L_{i, I} L_{j, I}}\right]^{1 / 4} \quad C_{\tilde{\chi}_{1}^{i j}}=\left(\alpha^{\prime}\right)^{1 / 4} \sqrt{2 \alpha^{\prime}} \prod_{I}\left[\frac{\alpha^{\prime}}{L_{i, I} L_{j, I}}\right]^{1 / 4}
\end{gathered}
$$

The factorization in the $t$ channel exhibits Yukawa couplings. After Poisson resummation we get $\mathscr{A}\left(\bar{\psi}_{0}, \psi_{0}, \chi_{0}, \bar{\chi}_{0}\right)=g_{\mathrm{op}}^{2} \alpha^{\prime} \psi_{0}(2) \cdot \chi_{0}(3) \bar{\psi}_{0}(1) \cdot \bar{\chi}_{0}(4) \int_{0}^{1} d x x^{\alpha^{\prime} s-1}(1-x)^{\alpha^{\prime} t-3 / 2} \prod_{I=1}^{3} \sum_{\tilde{m}_{I}, n_{I}} \frac{e^{-S_{\text {Lagr }}^{(l)}\left(\tilde{m}_{I}, n_{I}\right)}}{2 \pi \sqrt{I_{I}(x)}}$

where $I_{I}(x)$ are combinations of hypergeometric functions and $S_{\text {Lagr }}^{(I)}\left(\tilde{m}_{I}, n_{I}\right)$ is the classical action in "lagrangian" form. The limit $x \rightarrow 1$ has two kinds of contributions: one purely quantum, due to the expansion of $I_{I}(x)$, and one classical, due to the expansion of $t(x)$ in the action. The first three orders correspond to factorizations on the poles for the states $\phi_{0}, \phi_{1}$ and $\phi_{2}$. Imposing

$$
\mathscr{A}\left(\bar{\psi}_{0}, \psi_{0}, \chi_{0}, \bar{\chi}_{0}\right) \stackrel{t \rightarrow n a_{a b}^{1} / \alpha^{\prime}}{\longrightarrow}\left|Y_{n 00}\right|^{2} \psi_{0}(2) \cdot \chi_{0}(3) \frac{1}{t-n a_{a b}^{1} / \alpha^{\prime}} \bar{\chi}_{0}(4) \cdot \bar{\psi}_{0}(1)
$$

The Yukawa's extracted by factorization are given by

$$
\begin{aligned}
& \left|Y_{000}\right|=g_{\text {op }}(2 \pi)^{-3 / 4}\left[\Gamma_{1-a_{a b}^{1}, 1-a_{b c}^{1},-a_{c a}^{1}} \Gamma_{1-a_{a b}^{2}, 1-a_{b c}^{2},-a_{c a}^{2}} \Gamma_{-a_{a b}^{3},-a_{b c}^{3},-a_{c a}^{3}}\right]^{1 / 4} \prod_{I=1}^{3} \exp \left[-\frac{A_{\phi \psi \chi}^{(I)}}{2 \pi \alpha^{\prime}}\right] \\
& \left|Y_{100}\right|=\frac{\left|Y_{000}\right|}{\sqrt{a_{a b}^{1}}}\left[\Gamma_{1-a_{a b}^{1}, 1-a_{b c}^{1},-a_{c a}^{1}}\right]^{1 / 2} \sqrt{\frac{2 A_{\phi \psi \chi}^{(1)}}{\pi \alpha^{\prime}}} \\
& \left|Y_{200}\right|=\frac{\left|Y_{000}\right|}{\sqrt{2} a_{a b}^{1}} \Gamma_{1-a_{a b}^{1}, 1-a_{b c}^{1},-a_{c a}^{1}}\left|\frac{2 A_{\phi \psi \chi}^{(1)}}{\pi \alpha^{\prime}}-1\right|
\end{aligned}
$$

where $\Gamma_{a, b, c}=\frac{\Gamma(a) \Gamma(b) \Gamma(c)}{\Gamma(1-a) \Gamma(1-b) \Gamma(1-c)}$, and $A_{\phi \psi \chi}^{(I)}$ is the area of the triangle defined by the three points $f_{\psi, I}, f_{\chi, I}$ and $f_{\phi, I}$ in the torus $I$ given by $A_{\phi \psi \chi}^{(I)}=\frac{\sin \pi\left|a_{b c}^{I}\right| \sin \pi\left|a_{c a l}^{I}\right|}{2 \sin \pi\left|a_{a b}^{I}\right|} f_{\chi \psi, I}^{2}$. 
For the rest of the Yukawas $Y_{010}, Y_{001}$ etc we use supersymmetric Ward identities [1] and we get

$$
\begin{aligned}
& \left|Y_{010}\right|=\frac{\left|Y_{000}\right|}{\sqrt{a_{b c}^{1}}}\left[\Gamma_{1-a_{a b}^{1}, 1-a_{b c}^{1},-a_{c a}^{1}}\right]^{1 / 2} \sqrt{\frac{2 A_{\phi \psi \chi}^{(1)}}{\pi \alpha^{\prime}}}, \quad\left|Y_{001}\right|=\frac{\left|Y_{000}\right|}{\sqrt{1+a_{c a}^{3}}}\left[\Gamma_{-a_{a b}^{3},-a_{b c}^{3},-a_{c a}^{3}}\right]^{1 / 2} \sqrt{\frac{2 A_{\phi \psi \chi}^{(3)}}{\pi \alpha^{\prime}}} \\
& \left|Y_{020}\right|=\frac{\left|Y_{000}\right|}{\sqrt{2} a_{b c}^{1}} \Gamma_{1-a_{a b}^{1}, 1-a_{b c}^{1},-a_{c a}^{1}}\left|\frac{2 A_{\phi \psi \chi}^{(1)}}{\pi \alpha^{\prime}}-1\right|, \quad\left|Y_{002}\right|=\frac{\left|Y_{000}\right|}{\sqrt{2}\left(1+a_{c a}^{3}\right)} \Gamma_{-a_{a b}^{3},-a_{b c}^{3},-a_{c a}^{3}}\left|\frac{2 A_{\phi \psi \chi}^{(3)}}{\pi \alpha^{\prime}}-1\right|
\end{aligned}
$$

which completes the list of Yukawas involving one massive (1st and 2nd excitations) and two massless states.

\subsection{Yukawa couplings from amplitudes with massive external legs}

For Yukawas of more than one massive particle involved we have to start by 4-pt amplitudes with massive external legs. Following our strategy again, we fix the normalizations of the vertex operators from amplitudes that include gauge bosons, and we evaluate Yukawa couplings with more than one massive field.

Without loss of generality, we take the amplitude $\mathscr{A}\left(\bar{\psi}_{1}, \psi_{0}, \chi_{0}, \bar{\chi}_{0}\right)$ with one massive state $\bar{\psi}_{1}$ and three massless $\psi_{0}, \chi_{0}, \bar{\chi}_{0}$ :

$$
\begin{aligned}
\mathscr{A}\left(\bar{\psi}_{1}, \psi_{0}, \chi_{0}, \bar{\chi}_{0}\right)= & \frac{g_{\mathrm{op}}^{2}}{\sqrt{a_{b c}^{1}}} \alpha^{\prime} \psi_{0}(2) \cdot \chi_{0}(3) \bar{\psi}_{1}(1) \cdot \bar{\chi}_{0}(4) \\
& \times \int_{0}^{1} d x x^{\alpha^{\prime} s-1}(1-x)^{\alpha^{\prime} t-1} \prod_{I=1}^{3} \frac{2 \pi \sqrt{\alpha^{\prime}}}{L_{c, I} G_{1}^{(I)}(x)} \sum_{m_{I}} \frac{2 \pi \sqrt{\alpha^{\prime}} m_{1}}{\sqrt{I_{1}(x)} L_{c, I}} e^{-S_{\mathrm{Ham}}^{(I)}\left(m_{I}, n_{I}\right)}
\end{aligned}
$$

Similar to the amplitudes with massless external states, we perform a Poisson resummation over the indices $m_{I}$ and obtain

$$
\begin{aligned}
\mathscr{A}\left(\bar{\psi}_{1}, \psi_{0}, \chi_{0}, \bar{\chi}_{0}\right)= & \frac{g_{\mathrm{op}}^{2}}{\sqrt{a_{b c}^{1}}} \alpha^{\prime} \psi_{0}(2) \cdot \chi_{0}(3) \bar{\psi}_{1}(1) \cdot \bar{\chi}_{0}(4) \int_{0}^{1} d x x^{\alpha^{\prime} s-1}(1-x)^{\alpha^{\prime} t-3 / 2-a_{a b}^{1}} \\
& \times \frac{G_{1}^{(1)}(x)}{I_{1}(x)} \prod_{I=1}^{3} \frac{1}{\sqrt{2 \pi I_{I}(x)}} \sum_{\tilde{m}_{I}, n_{I}} \frac{\tilde{m}_{1} L_{c, 1}+f_{\chi \psi, 1}}{2 \pi \sqrt{\alpha^{\prime}}} e^{-S_{\text {Lagr }}^{(l)}\left(\tilde{m}_{I}, n_{I}\right)}
\end{aligned}
$$

In the limit $x \rightarrow 1$ the leading term is the massless pole due to the chiral exchange in the $(a, b)$ sector. We have fixed all the normalizations that appear in the amplitudes and we already know the Yukawa's $Y_{010}^{*} Y_{000}$, thus factorization in this channel can be used to check that normalizations are consistent. The subleading terms determine the Yukawa $Y_{110}$

$$
\left|Y_{110}\right|=\left|Y_{000}\right|\left|\frac{2 A_{\phi \psi \chi}^{(1)}}{\pi \alpha^{\prime}}-1\right| \frac{1}{\sqrt{a_{a b}^{1} a_{b c}^{1}}} \Gamma_{1-a_{a b}^{1}, 1-a_{b c}^{1},-a_{c a}^{1}}
$$


We can obtain the Yukawas $Y_{101}$ and $Y_{011}$ from two amplitudes with massive bosons in the external states. As for the massless case, we can use Ward identities to relate these to amplitudes with only fermions. The explicit expressions of the amplitudes read

$$
\begin{aligned}
\mathscr{A}\left(\bar{\chi}_{1}^{a c}, \chi_{0}^{c a}, \phi_{0}^{a b}, \bar{\phi}_{0}^{b a}\right) & =\frac{g_{\mathrm{op}}^{2}}{\sqrt{1+a_{c a}^{3}}} \alpha^{\prime} \bar{\chi}_{1}^{a c}(1) k_{4} \chi_{0}^{c a}(2) \int_{0}^{1} d x x^{\alpha^{\prime} s-1}(1-x)^{\alpha^{\prime} t-5 / 2-a_{b c}^{3} \times} \\
& \times \frac{G_{1}^{(3)}(x)}{I_{3}(x)} \prod_{I=1}^{3} \frac{1}{\sqrt{2 \pi I_{I}(x)}} \sum_{\tilde{m}_{I}, n_{I}} \frac{\tilde{m}_{3} L_{a, 3}+f_{\psi \phi, 3}}{2 \pi \sqrt{\alpha^{\prime}}} e^{-S_{\text {Lagr }}^{(I)}\left(\tilde{m}_{I}, n_{I}\right)} \\
\mathscr{A}\left(\bar{\phi}_{1}, \phi_{0}, \chi_{0}, \bar{\chi}_{0}\right) & =\frac{g_{\mathrm{op}}^{2}}{\sqrt{a_{a b}^{1}}} \alpha^{\prime} \bar{\chi}_{0}^{c b}(4) k_{2} \bar{\chi}_{0}^{b c}(3) \int_{0}^{1} d x x^{\alpha^{\prime} s-1}(1-x)^{\alpha^{\prime} t-5 / 2-a_{c a}^{1} \times} \\
& \times \frac{G_{1}^{(1)}(x)}{I_{1}(x)} \prod_{I=1}^{3} \frac{1}{\sqrt{2 \pi I_{I}(x)}} \sum_{\tilde{m}_{I}, n_{I}} \frac{\tilde{m}_{1} L_{b, 1}+f_{\chi \phi, 1}}{2 \pi \sqrt{\alpha^{\prime}}} e^{-S_{\text {Lagr }}^{(I)}\left(\tilde{m}_{I}, n_{I}\right)}
\end{aligned}
$$

The limit $x \rightarrow 1$ yields two new Yukawa's

$$
\begin{aligned}
& \left|Y_{011}\right|=\frac{\left|Y_{000}\right|}{\sqrt{a_{b c}^{1}\left(1+a_{c a}^{3}\right)}}\left[\Gamma_{1-a_{a b}^{1}, 1-a_{b c}^{1},-a_{c a}^{1}} \Gamma_{-a_{a b}^{3},-a_{b c}^{3},-a_{c a}^{3}}\right]^{1 / 2} \sqrt{\frac{2 A_{\phi \psi \chi}^{(1)}}{\pi \alpha^{\prime}} \frac{2 A_{\phi \psi \chi}^{(3)}}{\pi \alpha^{\prime}}} \\
& \left|Y_{101}\right|=\frac{\left|Y_{000}\right|}{\sqrt{a_{a b}^{1}\left(1+a_{c a}^{3}\right)}}\left[\Gamma_{1-a_{a b}^{1}, 1-a_{b c}^{1},-a_{c a}^{1}} \Gamma_{-a_{a b}^{3},-a_{b c}^{3},-a_{c a}^{3}}\right]^{1 / 2} \sqrt{\frac{2 A_{\phi \psi \chi}^{(1)}}{\pi \alpha^{\prime}} \frac{2 A_{\phi \psi \chi}^{(3)}}{\pi \alpha^{\prime}}}
\end{aligned}
$$

The amplitude $\mathscr{A}\left(\bar{\psi}_{1}, \psi_{0}, \chi_{0}, \bar{\chi}_{1}\right)$ allows us to determine the Yukawa's $Y_{111}$ and $Y_{211}$.

$$
\begin{aligned}
\mathscr{A}\left(\bar{\psi}_{1}, \psi_{0}, \chi_{0}, \bar{\chi}_{1}\right)= & \frac{g_{\mathrm{op}}^{2}}{\sqrt{a_{b c}^{1}\left(1+a_{c a}^{3}\right)}} \alpha^{\prime} \psi_{0}(2) \cdot \chi_{0}(3) \bar{\psi}_{1}(1) \cdot \bar{\chi}_{1}(4) \int_{0}^{1} d x x^{\alpha^{\prime} s-1}(1-x)^{\alpha^{\prime} t-1} \\
& \times \prod_{I=1}^{3} \frac{4 \pi^{2} \sqrt{\alpha^{\prime}}}{L_{c, I} G_{1}^{(I)}(x)} \sum_{m_{I}, n_{I}} \frac{\alpha^{\prime} m_{1} m_{3}}{\sqrt{I_{1}(x) I_{3}(x)} L_{c, 1} L_{c, 3}} e^{-S_{\text {Ham }}^{(I)}\left(m_{I}, n_{I}\right)}
\end{aligned}
$$

Once again the amplitude does not expose gauge boson exchange, since the sum over the lattice forbids it. To study the $t$ channel we perform the usual Poisson resummation

$$
\begin{aligned}
& \mathscr{A}\left(\bar{\psi}_{1}, \psi_{0}, \chi_{0}, \bar{\chi}_{1}\right)=\frac{\alpha^{\prime} g_{\mathrm{op}}^{2}}{\sqrt{a_{b c}^{1}\left(1+a_{c a}^{3}\right)}} \psi_{0}(2) \cdot \chi_{0}(3) \bar{\psi}_{1}(1) \cdot \bar{\chi}_{0}(4) \int_{0}^{1} d x x^{\alpha^{\prime} s-1}(1-x)^{\alpha^{\prime} t-5 / 2+a_{a b}^{2}} \\
& \times \frac{G_{1}^{(1)}(x) G_{1}^{(3)}(x)}{I_{1}(x) I_{3}(x)} \prod_{I=1}^{3} \frac{1}{\sqrt{2 \pi I_{I}(x)}} \sum_{\tilde{m}_{I}, n_{I}} \frac{\left(\tilde{m}_{1} L_{c, 1}+f_{\psi \chi, 1}\right)\left(\tilde{m}_{3} L_{c, 3}+f_{\psi \chi, 3}\right)}{4 \pi^{2} \alpha^{\prime}} e^{-S_{\text {Lagr }}^{(I)}\left(\tilde{m}_{I}, n_{I}\right)}
\end{aligned}
$$


Factorizing this amplitude we obtain the Yukawa's

$$
\begin{aligned}
& \left|Y_{111}\right|=\frac{\left|Y_{000}\right|}{\sqrt{a_{a b}^{1} a_{b c}^{1}\left(1+a_{c a}^{3}\right)}} \Gamma_{1-a_{a b}^{1}, 1-a_{b c}^{1},-a_{c a}^{1}} \Gamma_{-a_{a b}^{3},-a_{b c}^{3},-a_{c a}^{3}}^{1 / 2}\left|\frac{2 A_{\phi \psi \chi}^{(1)}}{\pi \alpha^{\prime}}-1\right| \sqrt{\frac{2 A_{\phi \psi \chi}^{(3)}}{\pi \alpha^{\prime}}} \\
& \left|Y_{211}\right|=\frac{\left|Y_{000}\right|}{\sqrt{2} a_{a b}^{1} \sqrt{a_{b c}^{1}\left(1+a_{c a}^{3}\right)}} \Gamma_{1-a_{a b}^{1}, 1-a_{b c}^{1},-a_{c a}^{1}}^{3 / 2} \Gamma_{-a_{a b}^{3},-a_{b c}^{3},-a_{c a}^{3}}^{1 / 2}\left|\frac{2 A_{\phi \psi \chi}^{(1)}}{\pi \alpha^{\prime}}-3\right| \sqrt{\frac{2 A_{\phi \psi \chi}^{(1)}}{\pi \alpha^{\prime}} \frac{2 A_{\phi \psi \chi}^{(3)}}{\pi \alpha^{\prime}}}
\end{aligned}
$$

\section{Conclusions}

In [1] we have evaluated the Yukawa couplings between light stringy states and SM fields. Our results can be used in order to built an effective field theory including SM fields and light stringy states and study specific decays. If the string scale is at the few $\mathrm{TeV}$ range, decays of such particles might be the first stringy effect which might be visible at LHC or future experiments.

\section{References}

[1] P. Anastasopoulos, M. Bianchi and D. Consoli, "Yukawa's of light stringy states," Fortsch. Phys. 65 (2017) 0110 [arXiv:1609.09299 [hep-th]].

[2] A. Sagnotti, Closed Strings and Their Open String Descendants, Phys. Rept. 184 (1989) 167-175.

[3] M. Bianchi and A. Sagnotti, The Partition Function of the SO(8192) Bosonic String, Phys. Lett. B211 (1988) 407.

[4] M. Bianchi and A. Sagnotti, THE GEOMETRY OF OPEN STRING PARTITION FUNCTIONS, in Theoretical Advanced Study Institute in Elementary Particle Physics: Particles, Strings and Supernovae (TASI 88) Providence, Rhode Island, June 5-July 1, 1988, 1988.

[5] G. Pradisi and A. Sagnotti, Open String Orbifolds, Phys. Lett. B216 (1989) 59.

[6] M. Bianchi and A. Sagnotti, Open Strings and the Relative Modular Group, Phys. Lett. B231 (1989) 389.

[7] J. Dai, R. G. Leigh, and J. Polchinski, New Connections Between String Theories, Mod. Phys. Lett. A4 (1989) 2073-2083.

[8] M. Bianchi and A. Sagnotti, On the systematics of open string theories, Phys. Lett. B247 (1990) 517-524.

[9] M. Bianchi and A. Sagnotti, Twist symmetry and open string Wilson lines, Nucl. Phys. $B 361$ (1991) 519-538.

[10] M. Bianchi, G. Pradisi, and A. Sagnotti, Planar duality in the discrete series, Phys. Lett. B273 (1991) 389-398.

[11] M. Bianchi, G. Pradisi, and A. Sagnotti, Toroidal compactification and symmetry breaking in open string theories, Nucl. Phys. B376 (1992) 365-386.

[12] C. Angelantonj, M. Bianchi, G. Pradisi, A. Sagnotti, and Ya. S. Stanev, Chiral asymmetry in four-dimensional open string vacua, Phys. Lett. B385 (1996) 96-102, [hep-th/9606169]. 
[13] J. Polchinski, Dirichlet Branes and Ramond-Ramond charges, Phys. Rev. Lett. 75 (1995) 4724-4727, [hep-th/9510017].

[14] M. Berkooz, M. R. Douglas, and R. G. Leigh, Branes intersecting at angles, Nucl. Phys. B480 (1996) 265-278, [hep-th/9606139].

[15] R. Blumenhagen, M. Cvetič, P. Langacker, and G. Shiu, Toward realistic intersecting D-brane models, Ann.Rev.Nucl.Part.Sci. 55 (2005) 71-139, [hep-th/ 0502005 ].

[16] R. Blumenhagen, B. Körs, D. Lüst, and S. Stieberger, Four-dimensional String Compactifications with D-Branes, Orientifolds and Fluxes, Phys.Rept. 445 (2007) 1-193, [hep-th/ 0610327 ].

[17] F. Marchesano, Progress in D-brane model building, Fortsch.Phys. 55 (2007) 491-518, [hep-th/0702094].

[18] M. Cvetič and J. Halverson, TASI Lectures: Particle Physics from Perturbative and Non-perturbative Effects in D-braneworlds, arXiv:1101.2907.

[19] L. E. Ibanez and A. M. Uranga, String theory and particle physics: An introduction to string phenomenology. Cambridge University Press, 2012.

[20] I. Antoniadis, E. Kiritsis, and T. Tomaras, A D-brane alternative to unification, Phys.Lett. $\mathbf{B 4 8 6}$ (2000) 186-193, [hep-ph/0004214].

[21] G. Aldazabal, L. E. Ibanez, F. Quevedo, and A. Uranga, D-branes at singularities: A Bottom up approach to the string embedding of the standard model, JHEP 0008 (2000) 002, [hep-th/0005067].

[22] F. Gmeiner, R. Blumenhagen, G. Honecker, D. Lüst, and T. Weigand, One in a billion: MSSM-like D-brane statistics, JHEP 0601 (2006) 004, [hep-th/ 0510170 ].

[23] P. Anastasopoulos, T. Dijkstra, E. Kiritsis, and A. Schellekens, Orientifolds, hypercharge embeddings and the Standard Model, Nucl.Phys. B759 (2006) 83-146, [hep-th/ 0605226 ].

[24] M. Cvetič, J. Halverson, and R. Richter, Realistic Yukawa structures from orientifold compactifications, JHEP 0912 (2009) 063, [arXiv : 0905 . 3379].

[25] M. Cvetič, J. Halverson, P. Langacker, and R. Richter, The Weinberg Operator and a Lower String Scale in Orientifold Compactifications, JHEP 1010 (2010) 094, [arXiv: 1001.3148 ].

[26] N. Arkani-Hamed, S. Dimopoulos, and G. Dvali, The Hierarchy problem and new dimensions at a millimeter, Phys.Lett. B429 (1998) 263-272, [hep-ph / 9803315$].$

[27] I. Antoniadis, S. Dimopoulos, and G. Dvali, Millimeter range forces in superstring theories with weak scale compactification, Nucl.Phys. B516 (1998) 70-82, [hep-ph/9710204].

[28] I. Antoniadis, N. Arkani-Hamed, S. Dimopoulos, and G. Dvali, New dimensions at a millimeter to a Fermi and superstrings at a TeV, Phys.Lett. B436 (1998) 257-263, [hep-ph/980 4398].

[29] E. Kiritsis and P. Anastasopoulos, The Anomalous magnetic moment of the muon in the D-brane realization of the standard model, JHEP 0205 (2002) 054, [hep-ph/ 0201295$].$

[30] I. Antoniadis, E. Kiritsis, and J. Rizos, Anomalous U(1)s in type 1 superstring vacua, Nucl.Phys. B637 (2002) 92-118, [hep-th/0204153].

[31] D. Ghilencea, L. Ibáñez, N. Irges, and F. Quevedo, TeV scale Z-prime bosons from D-branes, JHEP $\mathbf{0 2 0 8}$ (2002) 016, [hep-ph/ 0205083$].$ 
[32] P. Anastasopoulos, 4-D anomalous U(1)'s, their masses and their relation to 6-D anomalies, JHEP 0308 (2003) 005, [hep-th/0306042].

[33] P. Anastasopoulos, Anomalous U(1)s masses in nonsupersymmetric open string vacua, Phys.Lett. B588 (2004) 119-126, [hep-th/ 0402105$].$

[34] P. Burikham, T. Figy, and T. Han, TeV-scale string resonances at hadron colliders, Phys.Rev. D71 (2005) 016005, [hep-ph/0 411094 ].

[35] C. Corianò, N. Irges, and E. Kiritsis, On the effective theory of low scale orientifold string vacua, Nucl.Phys. B746 (2006) 77-135, [hep-ph/ 0510332$].$

[36] P. Anastasopoulos, M. Bianchi, E. Dudas, and E. Kiritsis, Anomalies, anomalous U(1)'s and generalized Chern-Simons terms, JHEP 0611 (2006) 057, [hep-th / 060522 5].

[37] P. Anastasopoulos, F. Fucito, A. Lionetto, G. Pradisi, A. Racioppi, and Y. Stanev, Minimal Anomalous U(1)-prime Extension of the MSSM, Phys.Rev. D78 (2008) 085014, [arXiv: 0804.1156 ].

[38] R. Armillis, C. Coriano, M. Guzzi, and S. Morelli, An Anomalous Extra Z Prime from Intersecting Branes with Drell-Yan and Direct Photons at the LHC, Nucl.Phys. B814 (2009) 156-179, [arXiv:0809.3772].

[39] F. Fucito, A. Lionetto, A. Mammarella, and A. Racioppi, Stueckelino dark matter in anomalous U(1)-prime models, Eur.Phys.J. C69 (2010) 455-465, [arXiv: 0811.1953 ].

[40] L. A. Anchordoqui, H. Goldberg, X. Huang, D. Lüst, and T. R. Taylor, Stringy origin of Tevatron Wjj anomaly, Phys.Lett. B701 (2011) 224-228, [arXiv:1104.2302].

[41] L. A. Anchordoqui, I. Antoniadis, H. Goldberg, X. Huang, D. Lüst, and T. Taylor, Z'-gauge Bosons as Harbingers of Low Mass Strings, Phys.Rev. D85 (2012) 086003, [arXiv: 1107 . 4309].

[42] L. A. Anchordoqui, I. Antoniadis, H. Goldberg, X. Huang, D. Lüst, T. Taylor, and B. Vlcek, LHC Phenomenology and Cosmology of String-Inspired Intersecting D-Brane Models, Phys.Rev. D86 (2012) 066004, [arXiv:1206.2537].

[43] E. Dudas and J. Mourad, String theory predictions for future accelerators, Nucl.Phys. $\mathbf{5 5 7 5}$ (2000) 3-34, [hep-th/9911019].

[44] E. Accomando, I. Antoniadis, and K. Benakli, Looking for TeV scale strings and extra dimensions, Nucl.Phys. B579 (2000) 3-16, [hep-ph/9912287].

[45] S. Cullen, M. Perelstein, and M. E. Peskin, TeV strings and collider probes of large extra dimensions, Phys.Rev. D62 (2000) 055012, [hep-ph/ 0001166 ].

[46] C. Burgess, J. Matias, and F. Quevedo, MSLED: A Minimal supersymmetric large extra dimensions scenario, Nucl.Phys. B706 (2005) 71-99, [hep-ph/ 0404135$].$

[47] D. Chialva, R. Iengo, and J. G. Russo, Cross sections for production of closed superstrings at high energy colliders in brane world models, Phys.Rev. D71 (2005) 106009, [hep-ph/0 03125 ].

[48] M. Cicoli, C. Burgess, and F. Quevedo, Anisotropic Modulus Stabilisation: Strings at LHC Scales with Micron-sized Extra Dimensions, JHEP 1110 (2011) 119, [arXiv: 1105.2107$].$

[49] D. Chialva, P. B. Dev, and A. Mazumdar, Multiple dark matter scenarios from ubiquitous stringy throats, Phys.Rev. D87 (2013), no. 6 063522, [arXiv:1211.0250].

[50] M. Bianchi and A. V. Santini, String predictions for near future colliders from one-loop scattering amplitudes around D-brane worlds, JHEP 0612 (2006) 010, [hep-th/ 0607224 ]. 
[51] L. A. Anchordoqui, H. Goldberg, S. Nawata, and T. R. Taylor, Jet signals for low mass strings at the LHC, Phys.Rev.Lett. 100 (2008) 171603, [arXiv:0712.0386].

[52] L. A. Anchordoqui, H. Goldberg, S. Nawata, and T. R. Taylor, Direct photons as probes of low mass strings at the CERN LHC, Phys.Rev. D78 (2008) 016005, [arXiv : 0804 . 2013].

[53] D. Lüst, S. Stieberger, and T. R. Taylor, The LHC String Hunter's Companion, Nucl.Phys. B808 (2009) 1-52, [arXiv:0807.3333].

[54] L. A. Anchordoqui, H. Goldberg, D. Lüst, S. Nawata, S. Stieberger, and T. Taylor, Dijet signals for low mass strings at the LHC, Phys.Rev.Lett. 101 (2008) 241803, [arXiv: 0808 . 0497].

[55] L. A. Anchordoqui, H. Goldberg, D. Lüst, S. Stieberger, and T. R. Taylor, String Phenomenology at the LHC, Mod.Phys.Lett. A24 (2009) 2481-2490, [arXiv: 0909.2216 ].

[56] D. Lüst, O. Schlotterer, S. Stieberger, and T. Taylor, The LHC String Hunter's Companion (II): Five-Particle Amplitudes and Universal Properties, Nucl.Phys. $\mathbf{B 8 2 8}$ (2010) 139-200, [arXiv:0908.0409].

[57] L. A. Anchordoqui, H. Goldberg, D. Lüst, S. Nawata, S. Stieberger, and T. Taylor, LHC Phenomenology for String Hunters, Nucl.Phys. B821 (2009) 181-196, [arXiv: 0904.3547 ].

[58] L. A. Anchordoqui, W.-Z. Feng, H. Goldberg, X. Huang, and T. R. Taylor, Searching for string resonances in $e^{+} e^{-}$and $\gamma \gamma$ collisions, Phys.Rev. D83 (2011) 106006, [arXiv: 1012.3466 ].

[59] W.-Z. Feng, D. Lüst, O. Schlotterer, S. Stieberger, and T. R. Taylor, Direct Production of Lightest Regge Resonances, Nucl.Phys. B843 (2011) 570-601, [arXiv: 1007.5254 ].

[60] Z. Dong, T. Han, M.-x. Huang, and G. Shiu, Top Quarks as a Window to String Resonances, JHEP 1009 (2010) 048, [arXiv: 1004.5441 ].

[61] D. Carmi, TeV Scale Strings and Scattering Amplitudes at the LHC, arXiv: 1109.5161.

[62] M. Hashi and N. Kitazawa, Detectability of the second resonance of low-scale string models at the LHC, JHEP 1303 (2013) 127, [arXiv: 1212 . 5372].

[63] L. A. Anchordoqui, I. Antoniadis, D.-C. Dai, W.-Z. Feng, H. Goldberg, et. al., String Resonances at Hadron Colliders, arXiv:1407.8120.

[64] D. Lüst and T. R. Taylor, Limits on Stringy Signals at the LHC, arXiv:1308.1619.

[65] D. Berenstein, TeV-Scale strings, arXiv:1401.4491.

[66] P. Anastasopoulos, M. Bianchi, and R. Richter, Light stringy states, JHEP 1203 (2012) 068, [arXiv:1110.5424].

[67] P. Anastasopoulos, M. Bianchi, and R. Richter, On closed-string twist-field correlators and their open-string descendants, arXiv:1110.5359.

[68] P. Anastasopoulos and R. Richter, Production of light stringy states, JHEP 12 (2014) 059, [arXiv:1408.4810].

[69] P. Anastasopoulos and R. Richter, Twisted state production, PoS CORFU2014 (2015) 116.

[70] P. Anastasopoulos and M. Bianchi, Revisiting light stringy states in view of the $750 \mathrm{GeV}$ diphoton excess, arXiv:1601.0758.

[71] P. Anastasopoulos, M. D. Goodsell, and R. Richter, Three- and Four-point correlators of excited bosonic twist fields, JHEP 10 (2013) 182, [arXiv:1305. 7166 ]. 
[72] R. Blumenhagen, L. Görlich, B. Körs, and D. Lüst, Noncommutative compactifications of type I strings on tori with magnetic background flux, JHEP 0010 (2000) 006, [hep-th/ 0007024 ].

[73] C. Angelantonj, I. Antoniadis, E. Dudas, and A. Sagnotti, Type I strings on magnetized orbifolds and brane transmutation, Phys.Lett. $\mathbf{B 4 8 9}$ (2000) 223-232, [hep-th/ 0007090 ].

[74] G. Aldazabal, S. Franco, L. E. Ibáñez, R. Rabadán, and A. Uranga, Intersecting brane worlds, JHEP 0102 (2001) 047, [hep-ph/ 0011132$].$

[75] G. Aldazabal, S. Franco, L. E. Ibáñez, R. Rabadán, and A. Uranga, D = 4 chiral string compactifications from intersecting branes, J.Math.Phys. 42 (2001) 3103-3126, [hep-th/0011073].

[76] S. Förste, G. Honecker, and R. Schreyer, Supersymmetric $Z(N) \times Z(M)$ orientifolds in 4-D with D branes at angles, Nucl.Phys. B593 (2001) 127-154, [hep-th/ 0008250 ].

[77] L. E. Ibáñez, F. Marchesano, and R. Rabádan, Getting just the standard model at intersecting branes, JHEP 0111 (2001) 002, [hep-th/ 0105155$].$

[78] M. Cvetič, G. Shiu, and A. M. Uranga, Three family supersymmetric standard - like models from intersecting brane worlds, Phys.Rev.Lett. 87 (2001) 201801, [hep-th/ 0107143$].$

[79] M. Cvetič, G. Shiu, and A. M. Uranga, Chiral four-dimensional N=1 supersymmetric type $2 A$ orientifolds from intersecting D6 branes, Nucl.Phys. B615 (2001) 3-32, [hep-th/ 0107166 ].

[80] G. Honecker, Chiral supersymmetric models on an orientifold of $Z(4) \times Z(2)$ with intersecting D6-branes, Nucl.Phys. B666 (2003) 175-196, [hep-th / 0303015 ].

[81] M. Cvetič, P. Langacker, T.-j. Li, and T. Liu, D6-brane splitting on type IIA orientifolds, Nucl.Phys. B709 (2005) 241-266, [hep-th/ 0407178$].$

[82] G. Honecker, Chiral N=1 4-D orientifolds with D-branes at angles, Mod.Phys.Lett. A19 (2004) 1863-1879, [hep-th/0407181].

[83] G. Honecker and T. Ott, Getting just the supersymmetric standard model at intersecting branes on the Z(6) orientifold, Phys.Rev. D70 (2004) 126010, [hep-th/ 0404055 ].

[84] R. Blumenhagen, F. Gmeiner, G. Honecker, D. Lüst, and T. Weigand, The Statistics of supersymmetric D-brane models, Nucl.Phys. B713 (2005) 83-135, [hep-th/ 0411173 ].

[85] R. Blumenhagen, M. Cvetič, F. Marchesano, and G. Shiu, Chiral D-brane models with frozen open string moduli, JHEP 0503 (2005) 050, [hep-th/ 05020 95].

[86] D. Bailin and A. Love, Towards the supersymmetric standard model from intersecting D6-branes on the Z-prime(6) orientifold, Nucl.Phys. B755 (2006) 79-111, [hep-th/ 0603172$].$

[87] M. Cvetič and P. Langacker, New Grand Unified Models with Intersecting D6-branes, Neutrino Masses, and Flipped SU(5), Nucl.Phys. B776 (2007) 118-137, [hep-th / 0607238 ].

[88] C.-M. Chen, T. Li, V. Mayes, and D. V. Nanopoulos, A Realistic world from intersecting D6-branes, Phys.Lett. B665 (2008) 267-270, [hep-th/ 0703280$]$.

[89] D. Bailin and A. Love, Almost the supersymmetric standard model from intersecting D6-branes on the Z(6)-prime orientifold, Phys.Lett. B651 (2007) 324-328, [arXiv: 0705.0646 ].

[90] F. Gmeiner and G. Honecker, Mapping an Island in the Landscape, JHEP 0709 (2007) 128, [arXiv:0708.2285]. 
[91] D. Bailin and A. Love, Constructing the supersymmetric Standard Model from intersecting D6-branes on the Z(6)-prime orientifold, Nucl.Phys. B809 (2009) 64-109, [arXiv: 0801 . 3385].

[92] F. Gmeiner and G. Honecker, Millions of Standard Models on Z-prime(6)?, JHEP 0807 (2008) 052, [arXiv:0806.3039].

[93] G. Honecker, M. Ripka, and W. Staessens, The Importance of Being Rigid: D6-Brane Model Building on $T^{6} / Z_{2} x Z_{6}^{\prime}$ with Discrete Torsion, Nucl.Phys. B868 (2013) 156-222, [arXiv: 1209.3010 ].

[94] G. Honecker and W. Staessens, D6-Brane Model Building and Discrete Symmetries on T 6/(Z2 $\times$ Z6 $\times \Omega R$ ) with Discrete Torsion, PoS Corfu2012 (2013) 107, [arXiv: 1303.6845 ]. 